\title{
Mechanisms for current-induced conductivity changes in a conducting polymer
}

\author{
$\mathrm{Xin} \mathrm{Xu}$ \\ Department of Electrical Engineering, Princeton University, Princeton, New Jersey 08544 \\ Richard A. Register \\ Department of Chemical Engineering, Princeton University, Princeton, New Jersey 08544 \\ Stephen R. Forrest ${ }^{\mathrm{a})}$ \\ Department of Electrical Engineering and Computer Science, University of Michigan, Ann Arbor, \\ Michigan 48109 and Department of Physics, University of Michigan, Ann Arbor, Michigan 48109
}

(Received 25 April 2006; accepted 22 August 2006; published online 4 October 2006)

\begin{abstract}
A layer of polyethylene dioxythiophene:polystyrene sulfonic acid (PEDT:PSS) spun onto the surface of an inorganic semiconductor forms a highly asymmetric rectifying junction when a small current is applied and can be permanently open circuited with application of a high current density. This allows the polymer/semiconductor junction to function as a write-once-read-many-times memory element. We use x-ray photoelectron spectroscopy and temperature induced conductivity measurements to study the morphological and chemical changes responsible for the large current-induced conductivity changes. It is found that by applying a large current to the organic-inorganic semiconductor rectifying heterojunction structure Au/PEDT:PSS/Si, the ratio of $\mathrm{PEDT}^{+}$to $\mathrm{PSS}^{-}$near the interface changes due to phase segregation in the presence of both high electric field $\left(>10^{5} \mathrm{~V} / \mathrm{cm}\right)$ and temperature. This leads to a decrease in film conductivity by up to six orders of magnitude from its value in the conductive state. (C) 2006 American Institute of Physics. [DOI: 10.1063/1.2358309]
\end{abstract}

Organic devices promise to revolutionize the extent and access to electronics by providing low-cost, flexible, large area, and lightweight components. During the last several years, a wide variety of devices based on polymers and small molecules has been demonstrated, including field effect transistors, light emitting diodes, and photovoltaic cells. ${ }^{1}$ Another important device, the organic electronic memory, has received comparatively little attention despite its important role in a wide range of electronic appliances. Recently, however, Möller et al. showed that an electronically written and addressed passive matrix write-once-read-many (WORM) times memory element can be realized by integrating a polymer fuse with a silicon diode. ${ }^{2,3}$ Subsequently, a simplified WORM memory structure was demonstrated by depositing the polymer directly onto a doped Si substrate, thereby eliminating the need for patterning and fabricating a $\mathrm{Si} p-i-n$ diode. ${ }^{4}$ The conducting polymer fuse in both structures employs polyethylene dioxythiophene:polystyrene sulfonic acid (PEDT:PSS), ${ }^{5}$ which undergoes an irreversible reduction in conductivity when exposed to a large (write) current. The $\mathrm{read} /$ write conductivity contrast ratio ("on/off ratio") is typically $>10^{5}$. Besides PEDT:PSS, more recently, other copolymers qualified for memory including, $N$-vinylcarbazole and Eu-complexed vinylbenzoate (PKEu) fluorene and chelated europium complex (PF8Eu). ${ }^{6,7}$

With the interest in WORM memory using PEDT:PSS, this work will focus on understanding the mechanism that leads to the extreme and permanent conductivity change of the polymer. Employing spectroscopic surface probes to examine the molecular changes that PEDT:PSS undergoes from the high to the low conductivity state, we conclude that the permanent conductivity decrease in PEDT:PSS is caused by

\footnotetext{
${ }^{a)}$ Electronic mail: stevefor@umich.edu
}

segregation of $\mathrm{PEDT}^{+}$and $\mathrm{PSS}^{-}$near the interface in the presence of a large current.

The first set of experiments employed $n$-doped Si (100) substrates $(1-5 \Omega \mathrm{cm})$ that were solvent cleaned and then deoxidized in $\mathrm{HF}: \mathrm{H}_{2} \mathrm{O}$ (1:1). A $70 \mathrm{~nm}$ thick layer of PEDT:PSS (1:2.5) (Ref. 5) was deposited onto the Si surface by spinning from an aqueous solution. To remove residual water, the polymer film was baked at $120^{\circ} \mathrm{C}$ for $1 \mathrm{~h}$ in a nitrogen filled glovebox $\left(<1 \mathrm{ppm} \mathrm{O}_{2}\right.$ and $\left.<1 \mathrm{ppm}_{2} \mathrm{O}\right)$. Gold contacts were thermally evaporated onto the PEDT:PSS surface through a shadow mask to form $50 \mathrm{~nm}$ thick, $5 \times 10^{-4} \mathrm{~cm}^{-2}$ metal contacts. After deposition, the conducting polymer surrounding the $\mathrm{Au}$ contacts was removed by reactive ion etching using $\mathrm{O}_{2}$ plasma at a flow rate of 50 SCCM (SCCM denotes cubic centimeters per minute at STP), a pressure of $100 \mathrm{mTorr}$, and a power of $50 \mathrm{~W}$ to prevent current spreading beyond the electrode perimeter. $\mathrm{X}$-ray photoelectron spectroscopy (XPS) data were acquired using a $150 \mu \mathrm{m}$ diameter focused probe beam of monochromatic $\mathrm{Al} K_{\alpha}$ radiation. The escape depth of the photoelectrons limits the analysis of compositional changes to within 50-100 $\AA$ of the PEDT:PSS surface.

Within $100 \mathrm{~ns}-1 \mu \mathrm{s}$ after the onset of a "switching current" of density from 10 to $100 \mathrm{~A} / \mathrm{cm}^{2}$, the polymer reaches a stable insulating state. ${ }^{2-4}$ To better understand the mechanisms leading to the current-induced conductivity changes, the samples were probed using XPS both prior to and following the current pulse. After application of current through one of the two samples under forward bias (corresponding to a positive potential at the $\mathrm{Au}$ electrode), the Au contact was removed using Scotch tape from both the switched and unswitched (control) samples.

The underside of the removed $\mathrm{Au}$ contact itself was first examined by XPS tuned to detect the presence of $\mathrm{O}, \mathrm{C}, \mathrm{S}$, and $\mathrm{Au}$. Negligible amounts of $\mathrm{C}$ and $\mathrm{O}$ were found on the 
TABLE I. Surface concentrations (at. \%) for PEDT:PSS in both its conducting (nonswitched) and insulating (switched) states.

\begin{tabular}{lcccccc}
\hline \hline \multicolumn{1}{c}{ Sample } & $\mathrm{C}$ & $\mathrm{O}$ & $\mathrm{Au}$ & $\mathrm{S}_{\text {tot }}{ }^{\mathrm{a}}$ & $\mathrm{S}_{\mathrm{PSS}}{ }^{\mathrm{b}}$ & $\mathrm{S}_{\text {PEDT }}{ }^{\mathrm{c}}$ \\
\hline Conducting state & $63 \pm 2$ & $24 \pm 2$ & $0.5 \pm 0.1$ & $8.3 \pm 0.2$ & $5.4 \pm 0.2$ & $2.9 \pm 0.1$ \\
Insulating state & $63 \pm 1$ & $24 \pm 1$ & $0.4 \pm 0.1$ & $8.2 \pm 0.1$ & $6.1 \pm 0.1$ & $2.1 \pm 0.1$ \\
\hline \hline
\end{tabular}

${ }^{\mathrm{a}} \mathrm{S}_{\text {tot }}$ is the total amount of sulfur detected in each device.

${ }^{\mathrm{b}} \mathrm{S}_{\mathrm{PSS}}$ represents the amount of sulfur from $\mathrm{PSS}^{-}$.

${ }^{\mathrm{c}} \mathrm{S}_{\mathrm{PEDT}}$ is the portion of sulfur from PEDT ${ }^{+}$. $\mathrm{S}_{\text {tot }}=\mathrm{S}_{\mathrm{PSS}}+\mathrm{S}_{\mathrm{PEDT}}$.

$\mathrm{Au}$, indicating that the peeling process removes the contact without pulling off the polymer surface layers. Next, examining the polymer surface, we find that the total elemental concentrations of $\mathrm{O}, \mathrm{C}$, and $\mathrm{S}$ both before and after switching are unchanged (see Table I). The atomic ratio of $\mathrm{O}, \mathrm{C}$, and $\mathrm{S}$ in the film is $3: 8: 1$, which is as expected from the PSS $^{-}$-rich PEDT:PSS molecular components. However, the relative concentration of S near the surface does, in fact, change by a significant amount (see Fig. 1 for the S $2 P$ spectral comparison both before and after switching). To accurately obtain the ratio $R$ of $\mathrm{PEDT}^{+}$to $\mathrm{PSS}^{-}$, we show the normalized $\mathrm{PSS}^{-}$ peak intensity in Fig. 1. The two sulfur peaks from PEDT ${ }^{+}$ are centered at 164.3 and $165.5 \mathrm{eV}$, whereas for PSS $^{-}$there is only a single peak at $168.5 \mathrm{eV}$, consistent with previous reports. ${ }^{8,9}$ After switching, there is no shift in binding energy for all sulfur peaks, indicating no change in the chemical environments of $\mathrm{S}$ for either of the two counterions. However, $R$ decreases near the Au/polymer interface after switching, as obtained from the atomic concentration of $\mathrm{S}$ from $\mathrm{PEDT}^{+}$and $\mathrm{PSS}^{-}$(see Table I) and from the $\mathrm{S} 2 P$ spectra in Fig. 1.

It is known that the conductivity of bulk PEDT:PSS is decreased by three orders of magnitude, from 1 to $0.001 \mathrm{~S} \mathrm{~cm}^{-1}$, as $R$ decreases ${ }^{5}$ from 0.4 to 0.2 . From our XPS results, we find that $R$ at the sample surface decreases from 0.6 to 0.4 (see Table I). However, the conductivity decrease on switching ${ }^{2-4}$ is typically $10-100$ times larger than the factor of 1000 anticipated for this magnitude of change in

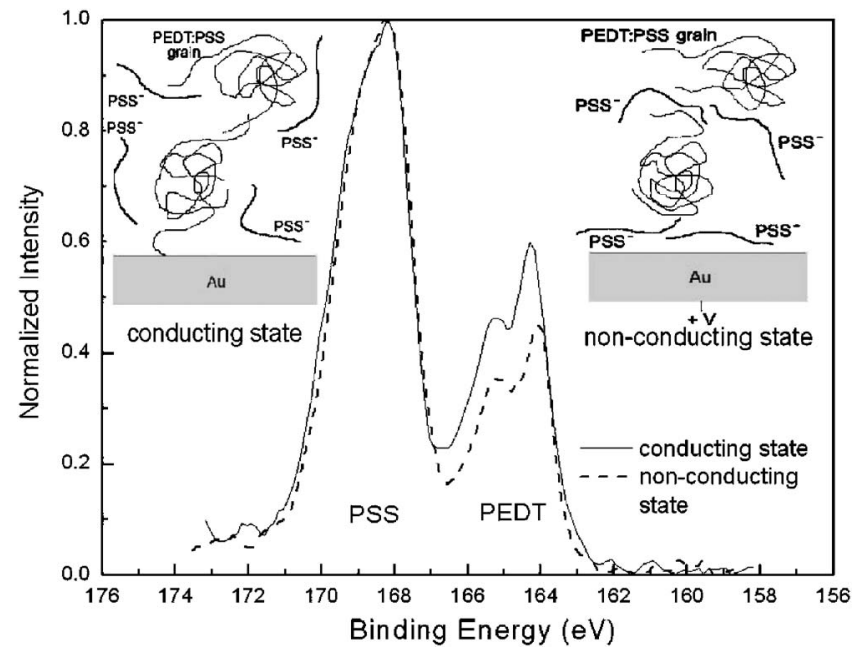

FIG. 1. Sulfur $2 P$ spectra at the cathode/polymer interface for a $n$-Si/PEDT:PSS/Au heterojunction device before (solid line) and after (dashed line) switching from the conductive to insulating state. The sulfur intensities for the PSS $^{-}$have been normalized for comparison. The left inset illustrates the morphology of $\mathrm{PEDT}^{+}$and $\mathrm{PSS}^{-}$before switching, whereas the right inset shows the phase segregation of two components following application of a large switching current and voltage.
$R$. We, therefore, infer that this unexpectedly large conductivity change results from morphological changes that accompany phase segregation in the near-surface region. When a PSS $^{-}$-rich PEDT:PSS dispersion is coated onto the substrate, a polymer film is formed with a granular morphology. ${ }^{8-10}$ That is, conducting PEDT:PSS particles are embedded in a $\mathrm{PSS}^{-}$-rich environment. ${ }^{10}$ In the presence of a large current (and hence large applied bias), the excess $\mathrm{PSS}^{-}$ chains migrate locally toward the surface under the resulting electric field. ${ }^{11}$ This results in the segregation of the conducting PEDT:PSS particles and the insulating PSS $^{-}$chains (see insets of Fig. 1) that prevents charge transport from the cathode to the nearby conducting PEDT:PSS grains and also blocks the conducting pathway between grains. This morphological change is anticipated to be activated by the temperature rise (approximately $\left.200^{\circ} \mathrm{C}\right)^{3}$ associated with the high current densities used in the switching process (see below). This model is consistent with the electric-field and temperature dependences of the switching phenomenon reported by Möller $e t a l^{3}$

To validate the existence of current-induced phase segregation near the cathode/polymer interface, we changed the substrate from $n$-doped $\mathrm{Si}$ to $10^{15} \mathrm{~cm}^{-3} p$-doped $\mathrm{Si}$. By changing the conductivity type, we are required to reverse the bias polarity required for switching. The XPS spectra near cathode/polymer interface are nearly identical to those shown in Fig. 1, with the decrease in $R$ is also approximately the same as in the case of $n$-type substrates, from 0.5 to 0.35 .

We observe that the Au contact is more easily peeled off from the polymer after application of a bias that induces conductivity switching. That is, prior to switching the $\mathrm{Au}$ cannot be cleanly delaminated from the polymer surface. This provides further evidence that morphological and compositional changes occur near the surface under conditions of current, voltage, and temperature used to switch the polymer from the conductive to nonconductive states.

To test whether compositional segregation occurs near the surface or throughout the film bulk, four identical samples of $70 \mathrm{~nm}$ thick PEDT:PSS (1:2.5) were switched under forward bias into their nonconducting states. Following switching, the $\mathrm{Au}$ contacts were removed as previously from three samples out of four, and the surface layers of the polymer were removed to a depth of $x=10,20$, or $45 \mathrm{~nm}$ by exposure to $\mathrm{O}_{2}$ plasma at a flow rate of $50 \mathrm{SCCM}$, a pressure of $100 \mathrm{mTorr}$, and a power of $50 \mathrm{~W}$ for 10,20 , or $40 \mathrm{~s}$, respectively, for the three samples. After etching, the Au contacts were once more redeposited onto the polymer surface.

Following the removal of the top $10 \mathrm{~nm}$ of material, the polymer returns to its high conductivity state and can once more be switched into the insulating state by reapplication of a current pulse, consistent with the behavior of pristine PEDT:PSS (see Fig. 2). However, this second switching re- 


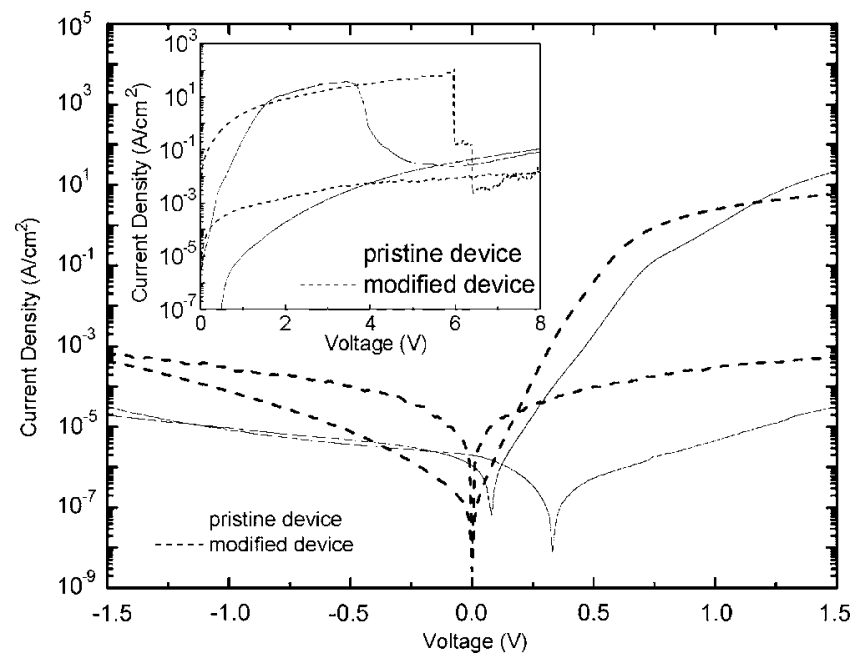

FIG. 2. Switching characteristics for a pristine $n$-Si/PEDT:PSS/Au device (solid line) and a modified device that is switched a second time following removal of the top $10 \mathrm{~nm}$ of the switched polymer (dashed line). Note the significant increase in switching current and voltage following the etching process. Inset shows the switching transient for both devices.

quires a larger voltage of $6 \mathrm{~V}$ and larger peak current density of $400 \mathrm{~A} / \mathrm{cm}^{2}$, compared with $4 \mathrm{~V}$ and approximately $10 \mathrm{~A} / \mathrm{cm}^{2}$ for the pristine device (see inset, Fig. 2). To examine for trends in switching versus depth, we compare the on/off ratios, conductivities after switching, switching voltages, and peak current densities leading to switching for the four samples in Table II. We find that the conductivity contrast is reduced, and switching occurs at higher currents and voltages as $x$ increases, with almost all the changes occurring within the first $20 \mathrm{~nm}$ from the polymer surface.

The conductivity change caused by segregation remains stable for periods of at least one month, as shown in the inset of Fig. 3. In contrast, the stability of the insulating state is reduced at elevated temperatures, supporting the hypothesis that the switching is at least partially thermally activated. ${ }^{2,3}$ After baking the switched device at $90{ }^{\circ} \mathrm{C}$ in vacuum for $1 \mathrm{~h}$, the switched device returns to the conductive state, from which it can be switched a second time (see Fig. 3). The thermal energy provided reintroduces disorder into the segregated film, returning the switched polymer to its original, conductive state.

In conclusion, we have found that the ratio of $\mathrm{PEDT}^{+}$to PSS $^{-}$changes near the cathode/polymer interface after applying a large switching current to a PEDT:PSS/Si heterojunction WORM memory element. The conductivity of the poly-

TABLE II. Switching properties as a function of removal of $x(\mathrm{~nm})$ of PEDT:PSS at the metal/polymer interface.

\begin{tabular}{|c|c|c|c|c|}
\hline & \multicolumn{4}{|c|}{$x(\mathrm{~nm})$} \\
\hline & 0 (pristine device) & 10 & 20 & 45 \\
\hline On/off ratio & $10^{6}$ & $10^{4}$ & $10^{3}$ & $10^{3}$ \\
\hline $\begin{array}{l}\text { Resistivity } \rho(\Omega \mathrm{cm}) \\
\text { (after switching) }\end{array}$ & $10^{12}$ & $10^{9}$ & $10^{10}$ & $10^{10}$ \\
\hline Switching voltage (V) & $3.5-4.5$ & 6 & 6 & 8 \\
\hline $\begin{array}{l}\text { Switching current } \\
\text { density }\left(\mathrm{A} / \mathrm{cm}^{2}\right)\end{array}$ & $10-50$ & 400 & 250 & 250 \\
\hline
\end{tabular}

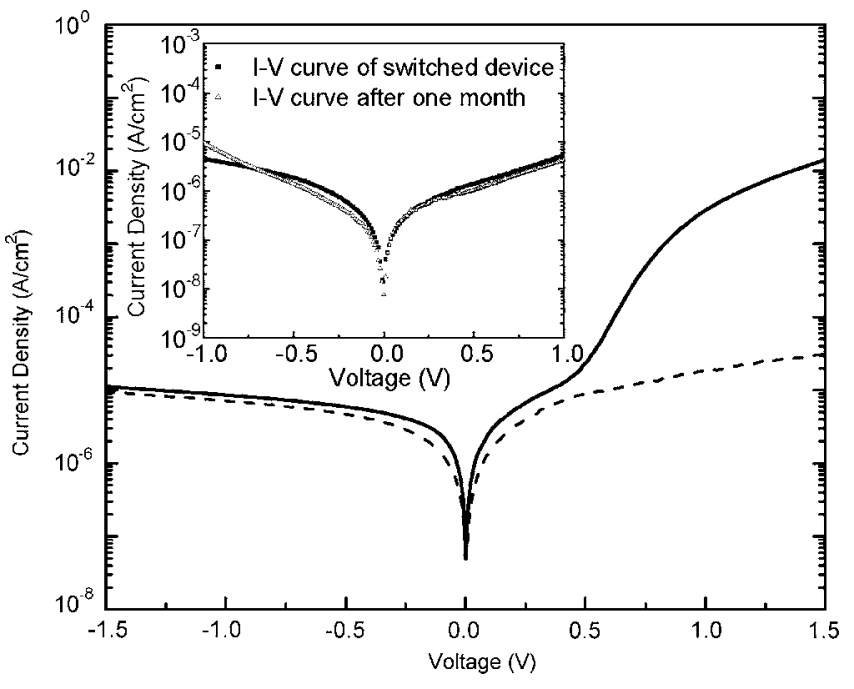

FIG. 3. Current-voltage characteristics for a switched $n$ $\mathrm{Si} / \mathrm{PEDT}$ : PSS/Au device after baking it at $90{ }^{\circ} \mathrm{C}$ in vacuum for $1 \mathrm{~h}$. The solid line shows data for a switched device after baking but before second switching. The dashed line is for the device after second switching. Inset: Current-voltage characteristics of the device immediately following switching (solid line) and one month later.

mer decreases due to phase segregation induced by the high switching current, which in turn subjects the polymer to an elevated temperature in the presence of a high applied electric field. This interpretation of the mechanism for switching is supported by the observation that elevated temperatures return a previously switched (and hence insulating) polymer back to its conductive state. These results suggest that optimal materials for use as fuses in WORM memories are those that can undergo irreversible morphological changes that are then stabilized at room temperature. These conditions, while apparently contradictory, can nevertheless result in long term stability under normal operating conditions, as has been demonstrated here.

The authors gratefully acknowledge the financial support of the National Science Foundation. They also thank Antoine Kahn, Jaehyung Hwang, Alan Wan, and Hongwei Zhang for helpful discussions.

${ }^{1}$ S. R. Forrest, Nature (London) 428, 911 (2004).

${ }^{2}$ S. Möller, C. Perlov, W. Jackson, C. Taussig, and S. R. Forrest, Nature (London) 426, 166 (2003).

${ }^{3}$ S. Möller, S. R. Forrest, C. Perlov, W. Jackson, and C. Taussig, J. Appl. Phys. 94, 7811 (2003).

${ }^{4}$ S. Smith and S. R. Forrest, Appl. Phys. Lett. 84, 5019 (2004).

${ }^{5}$ P. Baytron and H. C. Starck, Bayer Corp., Frankfurt, Germany.

${ }^{6}$ Q. D. Ling, Y. Song, S. J. Ding, C. Zhu, D. S. H. Chan, D.-L. Kwong, E.-T. Kang, and K.-G. Neoh, Adv. Mater. (Weinheim, Ger.) 17, 455 (2005).

${ }^{7}$ Y. Song, Q. D. Ling, C. Zhu, E. T. Kang, D. S. H. Chan, Y. H. Wang, and D.-L. Kwong, IEEE Electron Device Lett. 27, 154 (2006).

${ }^{8}$ G. Greczynski, T. Kugler, and W. R. Salaneck, Thin Solid Films 354, 129 (1999).

${ }^{9}$ G. Greczynski, T. Kugler, M. Keil, W. Osikowicz, M. Fahlman, and W. R. Salaneck, J. Electron Spectrosc. Relat. Phenom. 121, 1 (2001).

${ }^{10}$ X. Crispin, S. Marciniak, W. Osikowicz, G. Zotti, A. W. Denier van der Gon, F. Louwet, M. Fahlman, L. Groenendaal, F. De Schryver, and W. R. Salaneck, J. Polym. Sci., Part B: Polym. Phys. 41, 2561 (2003).

${ }^{11}$ S. J. Martin, R. A. L. Jones, M. Geoghegan, A. M. Higgins, I. Grizzi, J. J. M. Halls, S. Kirchmeyer, and R. M. Dalgleish, Phys. Rev. B 71, 081308 (2005). 